\title{
The Effect of the Width and Number of Gaps on the Characteristics of Swirl Flow Induced Naturally inside Split Channel Using Hot Air Inlet
}

\author{
Safia R. Al Atresh, Ahmad S. Sharifian, and Andrew P. Wandel
}

\begin{abstract}
Split channels are commonly employed in research laboratories to generate fire whirls. The purpose of this computational study is to investigate the effects of the width and number of side gaps on the performance of the split channel when hot air enters at the base of the channel instead of using a fire. Three cylindrical channels are modeled. The first channel includes two identical slots, the second channel has only one gap with a width double that of the first channel, and the third channel comprises one gap identical to that of the first channel. Comparisons between the results of the first and the second channel provide the assessment of the impacts of the number of gaps on the performance of the split channel. Moreover, the comparison between the results of the second and third channels shows the effect of changing the width of the gap. The combined (overall) effects of the number and the width of the gaps on the characteristics of the flow within the channel are also evaluated by comparing the results of the first and the third channel. The results show that increasing the number of the slots without increasing the total area of the gaps leads to an increase in centerline vorticity within the channel. In addition, the inlet velocity of hot air from the base and entrainment ratio decreases with the increase of the number of the side slots. Increasing the width of the gap without changing the number of the slots reduces the entrainment velocity, the inlet velocity, but the entrainment mass flow rate and centerline vorticity increase. The entrainment mass flow rate centerline vorticity and entrainment ratio increases by the increase in the number of identical gaps and the increase of gap width whereas the entrainment velocity and the inlet velocity decrease.
\end{abstract}

Index Terms - Split channel, number of gaps, width of gap, entrainment velocity.

\section{INTRODUCTION}

The fire whirl is a phenomenon that occurs rarely but is a potentially catastrophic form of fire. It can be induced within a split channel when the fire is located at its base. The split channel can also be used to generate swirling flow using hot air flow instead of a fire [1]. The interaction between the vertical component of the rising hot air and the tangential component of the entrainment velocity creates the swirling flow within the chamber.

In addition, such a configuration is simple and low-cost. It can also be used as part of fuel saving technology to induce swirl flow and enhance the mixing process of fuel and air

Manuscript received December 9, 2013; revised April 2, 2014. This work was supported financially by the Libyan government and the Computational Engineering and Science Research Centre (CESRC) at USQ.

S. R. Al Atresh is with Computational Engineering and Science Research Centre, the School of Mechanical and Electrical Engineering, University of Southern Queensland, Toowoomba, West St. Australia (e-mail: w0091920@usq.edu.au). within internal-combustion engines or as a split chimney. A fuel saver such as the Hiclone creates a swirling tornado effect in the airflow into the intake manifold and combustion chamber of any engine. This enhances the efficient burning of the fuel, improving fuel economy, creating more power, and reducing harmful emissions. The Hiclone's swirling effect makes fuel particles finer and allows up to $85 \%$ of the fuel to be burned, as opposed to about $60 \%$ in the best conventional systems [2]. This stationary swirl configuration can dilute the pollutants before they are released to the atmosphere [3] and it may accelerate the plume of existing pollutants from a chimney to be emitted higher in the atmosphere. The split channel with one gap is expected to be used for the same purposes, especially for accelerating the plume according to the results from this study.

Many studies have investigated fire whirl generation using a stationary vertical cylindrical split channel with one and two gaps. A vertical cylindrical channel, which was slightly warped into a spiral shape with one gap only, was used to generate a fire-whirl in a laboratory [4]. This demonstrated that one gap is sufficient to circulate the fire plume within a cylindrical channel. In reference [5], experimental work conducted using a split channel with 2 gaps discovered that the centerline vorticity is higher at the base. Furthermore, the entrainment velocity decreased continually with increasing width of the gap. Reference [6] addressed the modeling of a generated fire whirl within the channel and captured the essence of the fluid dynamics of flame-swirl interactions. A computational fluid dynamics (CFD) study using Fluent produced a fire whirl within a split channel with two side gaps [7].

A numerical study induced fire whirls on a vertical square shaft with one and two lateral gaps [8]. They reported that a fire whirl was observed experimentally, but they failed to demonstrate it numerically due to insufficient angular momentum using one gap. Reference [9] addressed the effect of increased width of the gap on inducing a virtual fire whirl using a similar geometry to that reported in reference [8]. They concluded that fire whirls would not be induced if the gap width is too small or too big. No previous CFD study is available to model swirl flow within a cylindrical split channel with one gap in the case of using a fire or hot air flow. The researchers in reference [10] generated a fire whirl within a square channel with four gaps. They reported that the entrainment velocity along the gap is higher at the base and it decreased with the increase of gap width. Researchers in reference [11] produced virtually fire whirl within a square duct with four corner gaps and they confirmed the results of reference [10]. Reference [12] showed the possibility to 
generate fire whirl inside a square channel with only one gap having width of $0.036 \mathrm{~m}$ using different types of fuel. They also used an electric heater as a heat source and smoke pellets to visualize the flow movements while retaining the same gap width. They found that the electrical heater could not produce swirl flow when the electrical power was less than $1.5 \mathrm{~kW}$.

Recent studies show that the split channels with two side slots, and conventional channels integrated with split caps are able to generate swirling flow using hot air flow instead of a fire pool. Numerical modeling has been performed [3] to show that a split channel can produce swirling flow and dilute the inlet flow from the base by $50 \%$ in a low-wind weather condition. In reference [13], the author addressed the effect of wide range of wind speeds on the performance of a split chimney and reported that the discharge of the chimney increases with the increase of wind speed. Reference [1] studied the effect of inlet static temperature and inlet velocity on the strength of produced swirl flow in a stagnant atmosphere. The swirling flows could be produced using hot air instead of burning fuel regardless of the temperature and velocity of the inlet air. However, it was reported that the swirl strength decreases with the decrease of the temperature and the velocity of the inlet air. It was also found that the axial velocity of the swirling flow decreases along the height of the channel whereas it reaches its maximum at the mid height of the channel in case of fire-whirls. Reference [14] reported the results from modeling a conventional chimney integrated with a split gap at its top to avoid side leakage. They found that the split gap generates swirl flow and the dilution increases linearly with the height of the gap.

In the current work, CFD is conducted to discover the effects of the number and width of gaps on the centerline axial vorticity, the entrainment velocity and entrainment mass flow rate, inlet axial velocity, inlet mass flow rate, inlet suction pressure, and entrainment ratio within a cylindrical split channel using hot air as the heat source. The ratio of entrainment mass flow to the inlet mass flow rate represents the dilution and the vorticity is relevant to the quality of the mixing of the inlet and entrainment flows. Three cylindrical split channels have been modeled with the same geometry with the differences being the gap of width and number of gaps. The first channel utilizes two identical slots, the second channel has only one gap with a width twice that of the first channel, and the third channel comprises one gap identical to that of the first channel. The main objectives of this paper are to evaluate the impacts of the number and the width of the gaps on the performance of modeled configurations.

\section{Methodology}

\section{A. CFD Modelling}

A commercial CFD solver (ANSYS-CFX) was used to model the three configurations. Figure 1a shows the top view of all three models. The first configuration, denoted $2 \mathrm{G} 2 \mathrm{~cm}$, has two identical side gaps and its dimensions are similar to those presented in references [1], [7] (see Table I and Fig. 1 for details). The internal diameters of the two halves of the split channels are $d c_{1}$ and $d c_{2}$, the wall thickness is $e$, the width of the gap is $W_{g}$, and the diameter of the inlet hot air is $d_{\text {in }}$ (see Fig. 1b). The second and third configurations, denoted $1 \mathrm{G} 4 \mathrm{~cm}$ and $1 \mathrm{G} 2 \mathrm{~cm}$ respectively, have only one side gap and the gap is generated in the same way as presented in reference [4]. The gap width of the second channel is $0.04 \mathrm{~m}$, which equals the total gap width of the first channel. The gap width of the third configuration is $0.02 \mathrm{~m}$, similar to the width of each gap in the first configuration (see Fig. 1a and Table I for details). The wind speed, ambient temperature, inlet temperature and inlet total hot air pressure are similar for all cases and equal to zero, $25^{\circ} \mathrm{C}, 1300^{\circ} \mathrm{C}$ and 1 atm, respectively.

The air is treated as an ideal gas with variable thermodynamic properties. The walls of the channels are modelled as smooth and adiabatic with no-slip conditions. Thermal radiation has not been modelled. The gravity is in the negative direction of the height (negative y-direction in Fig. 1). The ambient pressure is represented using the following equation [15]

$$
p=p_{o} \exp \left(-g \cdot y / R \cdot T_{0}\right)
$$

where $p$ represents static pressure, $p_{0}$ is the reference pressure (101325 Pa), $y$ is height with respect to the reference point, $R$ is the ideal gas constant for air $(287.1 \mathrm{~J} / \mathrm{kg} / \mathrm{K})$ and $T_{0}$ is the reference ambient temperature $(298.15 \mathrm{~K})$.

TABLE I: DiMENSIONS OF THE CONFIGURATIONS MODELLED

\begin{tabular}{ccccccc}
\hline \hline Channel & $d c_{1}$ & $d c_{2}$ & $W_{g}$ & $d_{\text {in }}$ & $H_{c}$ & $e$ \\
& $\mathrm{~m}$ & $\mathrm{~m}$ & $\mathrm{~m}$ & $\mathrm{~m}$ & $\mathrm{~m}$ & $\mathrm{~m}$ \\
\hline $2 \mathrm{G} 2 \mathrm{~cm}$ & 0.2 & 0.2 & 0.02 & 0.03 & 1 & 0.01 \\
$1 \mathrm{G} 4 \mathrm{~cm}$ & 0.2 & 0.25 & 0.04 & 0.03 & 1 & 0.01 \\
$1 \mathrm{G} 2 \mathrm{~cm}$ & 0.2 & 0.23 & 0.02 & 0.03 & 1 & 0.01 \\
\hline \hline
\end{tabular}

All three split channels are located at the centre of the base of a large cubical domain. The base of the domain is modelled as a wall, while the other five sides are treated as an opening because the pressure is known but the direction of flow is unknown. For the openings, in the case of inflow the defined value of pressure is considered as static pressure, but for outflow it is taken to be total pressure.

The governing equations implemented in the simulations are the continuity, Navier-Stokes, and thermal energy equations. The fully-buoyant model was utilized because of the large temperature differences between the inlet at the base $\left(1300^{\circ} \mathrm{C}\right)$ and ambient $\left(25^{\circ} \mathrm{C}\right)$. The shear stress transport turbulence model (SST) has been selected in the present study as recommended in [16]; and the inlet turbulent intensity (TI) at the base is the same as the initial TI of the domain $(5 \%)$.

A steady-state solver was used with high-order discretization schemes. The convergence criteria were set to $1 \times 10^{-5}$ for all variables based on RMS residuals. The modeling was conducted using the high performance computing facility (HPC) at University of Southern Queensland. Each node on the USQ HPC cluster has a 2.7 GHz AMD Opteron CPU and 16 GB DDR2-667 memory. Each simulation was conducted using 4 nodes on the cluster.

The setup has been verified through two techniques. In the first technique, the domain size was increased to investigate the possible impacts of the domain size on the results for the first configuration only, then applied to the other two 
configurations. The base domain size $(4 \mathrm{~m} \times 4 \mathrm{~m} \times 4 \mathrm{~m})$ was increased by $50 \%, 100 \%, 150 \%$ and $200 \%$ and it was found that the entrained velocity, centerline axial vorticity, mass flow rate, and inlet velocity change by less than $2 \%$ on average when comparing the $150 \%$ and $200 \%$ increases in domain size. As a result, the $10 \mathrm{~m} \times 10 \mathrm{~m} \times 10 \mathrm{~m}$ size $(150 \%$ increase) was chosen as the resolved domain size. The second technique was employed to make sure the results are independent of the mesh. The results show that minimum required number of resolved elements to reach converged and independent mesh results are 2.29, 2.08, and 2.76 million elements for the first, second and third configuration, respectively. The CFD models neglect the modelling of smoke particles and their effect on radiant heat transfer.
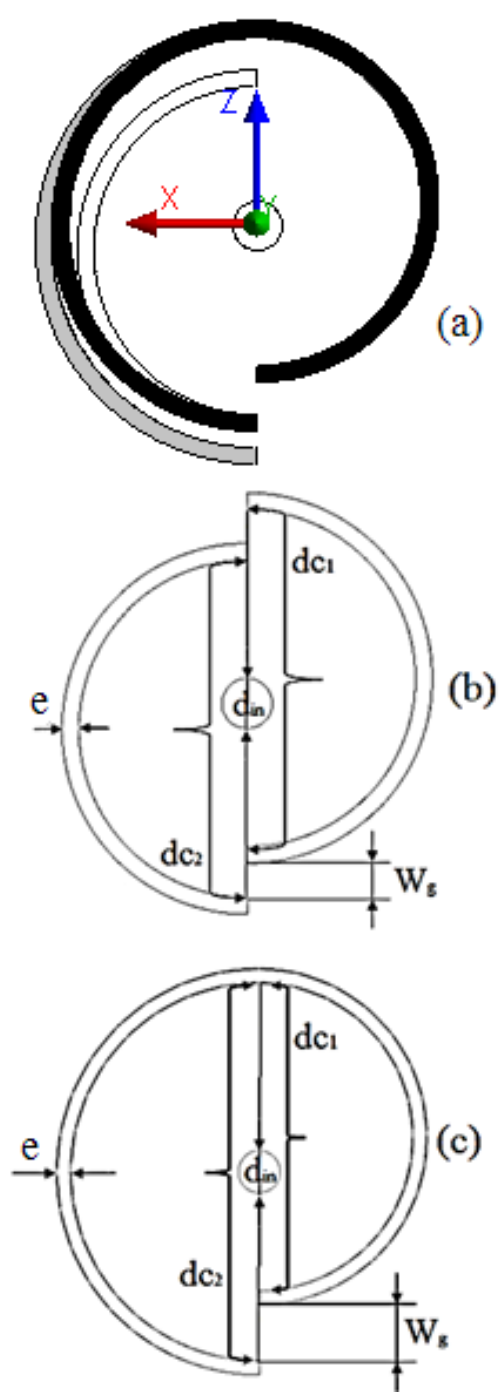

Fig. 1. Top view of models in this study. (a) Top view of all three models, (b) Top view of first model, (c) Top view of second model.

\section{B. Experimental Visualization}

Fig. 2 (a) shows the experimental setup where split channel with one gap was made in a manner similar to reference [4]. The set-up comprised an electrical heater, cylindrical volume, flat piece of circular wood, smoke machine, and split channel with one gap. The electrical heater with $1.6 \mathrm{~kW}$ was used as the heat source. A cylindrical volume was placed $0.02 \mathrm{~m}$ above the heater to allow entrainment through it. This volume has a port at the top with a $0.05 \mathrm{~m}$ diameter and side port with $0.02 \mathrm{~m}$ diameter. The top port is to induce the hot air plume, and side port to introduce the smoke. The piece of circular wood ( $0.02 \mathrm{~m}$ thick, $0.4 \mathrm{~m}$ diameter) has a central port of $0.03 \mathrm{~m}$ diameter and is located above the cylindrical volume to decrease the diameter of the port and to act as thermal insulation. The split cylinder with one gap $(0.03 \mathrm{~m}$ width of gap, $0.15 \mathrm{~m}$ height) is then located on the circular wood, where the port is in the centre of the split cylinder. Fig. 2(b) displays the visible image of the smoke $\left(d_{\text {in }}=3 \mathrm{~cm}\right)$ with spin.

\section{RESULTS AND DISCUSSION}

\section{A. Swirl Visualization}

The values of axial vorticity indicate that generating swirl flow within a one gap cylindrical channel is possible and the swirl visualization confirms this. Although the researchers in [12] could not generate swirl flow when using a hot surface, the cylindrical split channel in this study could induce swirling smoke and that is probably due to the different set up of the current study. The researchers in [12] made the heater cover the entire cross-sectional area of the square channel and the smoke pellet was too long thereby decreasing the possibility of rotating the smoke using one gap. In the current study, the heated air entered through the central portion of the channel, leaving space inside the channel for entrainment and circulation.

\section{B. Effects of Number of the Gaps on Swirl Flow Characteristics}

To know the real effect of number of gaps, the results of the $2 \mathrm{G} 2 \mathrm{~cm}$ model and the $1 \mathrm{G} 4 \mathrm{~cm}$ model are compared. The $2 \mathrm{G} 2 \mathrm{~cm}$ model has 2 gaps of $2 \mathrm{~cm}$ width, and the $1 \mathrm{G} 4 \mathrm{~cm}$ model has one gap of $4 \mathrm{~cm}$ width. The two models have equal gap area but the number of gaps is different.

In both channels, the maximum entrainment velocity occurs near the base of the split channels and then decreases as the height increases (see Fig. 3). The average entrainment velocity and the total entrainment mass flow rate increases slightly with a decrease in number of gaps (see Table II) and this increase occurs along the entire height of the gap (see Fig. $3)$.

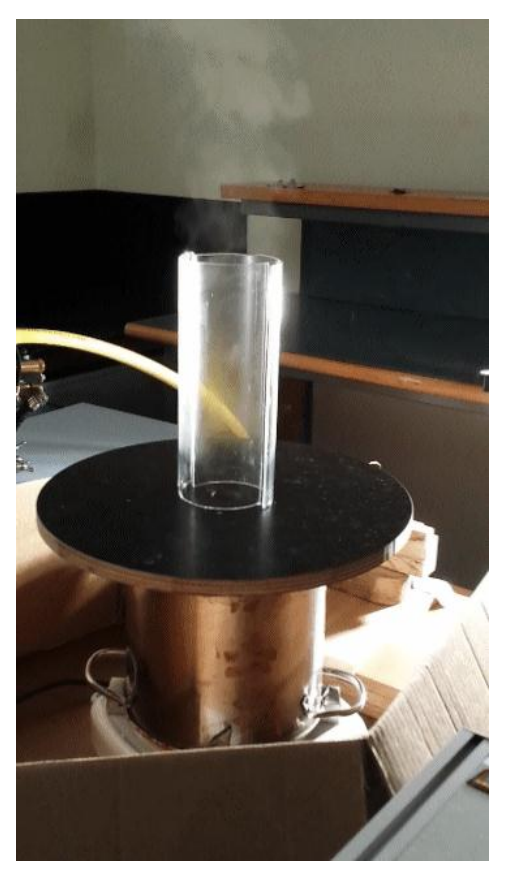

Fig. 2(a). Experimental set-up for swirl visualization. 


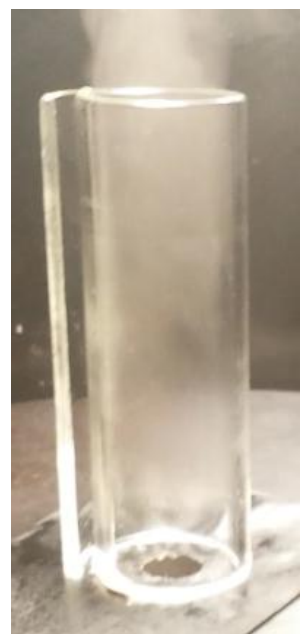

Fig. 2(b). Swirl smoke within channel with one slot only.

Due to the greatest difference in density between the ambient air and swirl hot plume occurring at the base, the maximum entrainment velocity occurs there. This difference decreases along the height as density differences decrease along the channel leading to reductions in the suction of air and consequently the entrainment velocity. In addition, the slight differences in the centerline pressure, and consequently the pressure gradients between the internal and surrounding ambient conditions, cause the slight difference in the entrainment velocity. Furthermore, for $1 \mathrm{G} 4 \mathrm{~cm}$, the slight increase of entrainment mass flow rate is because of the slight increase of entrainment velocity.

TABLE II: AVERAGE ENTRAINMENT VELOCITY U AND TOTAL ENTRAINMENT MASS FlOW RATE FOR THE THREE CONFIGURATIONS

\begin{tabular}{ccc}
\hline Channel & $\begin{array}{c}\text { Average Entrainment velocity u } \\
\mathrm{m} / \mathrm{s}\end{array}$ & $\begin{array}{c}\text { Total entrainment mass } \\
\text { flow rate } \\
\mathrm{kg} / \mathrm{s}\end{array}$ \\
\hline $2 \mathrm{G} 2 \mathrm{~cm}$ & 0.3690 & 0.01863 \\
$1 \mathrm{G} 4 \mathrm{~cm}$ & 0.4143 & 0.02050 \\
$1 \mathrm{G} 2 \mathrm{~cm}$ & 0.5910 & 0.01493 \\
\hline \hline
\end{tabular}

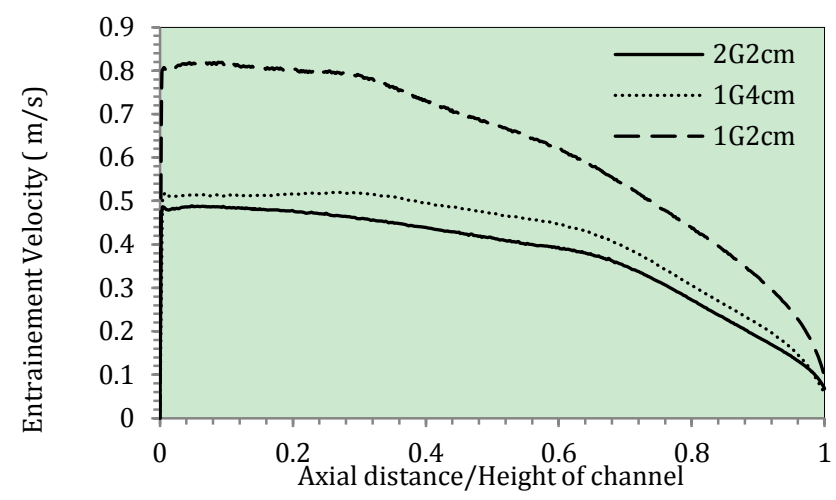

Fig. 3. Entrainment velocities along the gap for all three channels.

Fig. 4 indicates that centerline vorticity increases when the number of the gaps increases even though the entrainment mass flow rate of the $2 \mathrm{G} 2 \mathrm{~cm}$ channel is lower than the $1 \mathrm{G} 4 \mathrm{~cm}$ channel. There are two possible explanations which are: a more even distribution of the entrained flow in the $2 \mathrm{G} 2 \mathrm{~cm}$ channel than the $1 \mathrm{G} 4 \mathrm{~cm}$ channel, and the decrease of the inlet mass flow rate at the base of the $2 \mathrm{G} 2 \mathrm{~cm}$ channel. It is clear that two symmetrical gaps allow reversed currents of ambient air along the gap and this can increase the vorticity inside the channel. In addition, the lower inlet mass flow rate can allow slower flow inside the channel, creating more opportunity for the entrained air to penetrate the swirl flow and affect the centerline vorticity.

The inlet axial velocity and mass flow rate decrease slightly as the number of gaps increases because the average inlet static suction decreases slightly with the increase in the number of gaps (see Table III).

Additionally, the entrainment ratio decreases slightly with the increase of the number of the gaps and this is mainly because of the decrease of entrainment mass flow rate (see Table IV).

\section{Effects of Width of the Gap (Overall Area of the Gap) on Swirl Flow Characteristics}

To examine the effect of gap width, the results of the $1 \mathrm{G} 4 \mathrm{~cm}$ and the $1 \mathrm{G} 2 \mathrm{~cm}$ channels are compared.

The narrowing of the gap leads to a significant increase of the average entrainment velocity, and decrease of the total entrainment mass flow rate (see Table II). As shown also in Fig. 3, the entrainment velocity along the gap for the $1 \mathrm{G} 2 \mathrm{~cm}$ channel is higher than the $1 \mathrm{G} 4 \mathrm{~cm}$ channel.

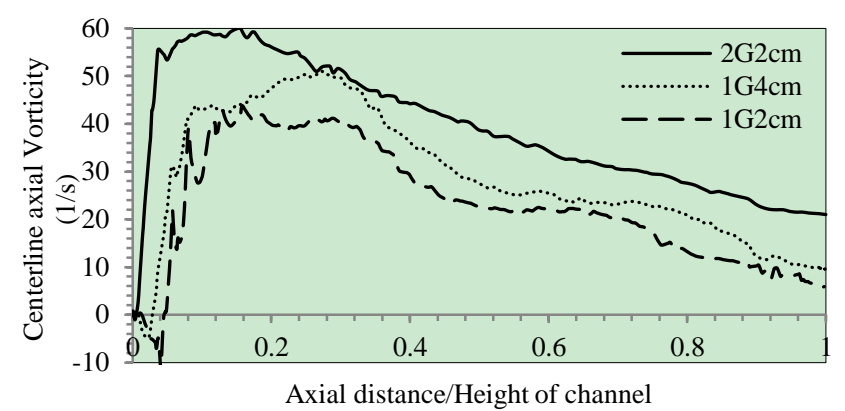

Fig. 4. Axial vorticity along the centreline of plume into each of the three channels.

TABLE III: AVERAGE INLET AXIAL VELOCITY, INLET STATIC SUCTION PRESSURE \& MASS FlOW RATE FOR THE THREE CHANNELS

\begin{tabular}{cccc}
\hline \hline Channel & $\begin{array}{c}\text { Average inlet } \\
\text { axial velocity }\end{array}$ & $\begin{array}{c}\text { Average inlet mass } \\
\text { flow rate }\end{array}$ & $\begin{array}{c}\text { Inlet static } \\
\text { suction } \\
\text { pressure }\end{array}$ \\
\hline $2 \mathrm{G} 2 \mathrm{~cm}$ & $\mathrm{~m} / \mathrm{s}$ & $\mathrm{kg} / \mathrm{s}$ & $\mathrm{Pa}$ \\
$1 \mathrm{G} 4 \mathrm{~cm}$ & 3.80 & $6.29 \times 10^{-4}$ & -2.01 \\
$1 \mathrm{G} 2 \mathrm{~cm}$ & 4.77 & $7.78 \times 10^{-4}$ & -2.82 \\
\hline \hline
\end{tabular}

These results can only be explained using the pressure drop within the split channel. A low pressure region is created inside the $1 \mathrm{G} 2 \mathrm{~cm}$ channel (see Fig. 5) that increases the pressure drop between the interior of the channel and the surrounding ambient fluid. The average entrainment velocity also increases because of the higher entrainment velocity along the gap. Furthermore, although the entrainment velocity of $1 \mathrm{G} 2 \mathrm{~cm}$ channel is higher than $1 \mathrm{G} 4 \mathrm{~cm}$, the smaller cross-sectional area of $1 \mathrm{G} 2 \mathrm{~cm}$ channel results in a smaller entrainment mass flow rate.

According to Fig. 4, the centerline vorticity decreases with the decrease of the width of gap along the channel. This shows how the magnitudes of centerline vorticity are affected by the different value of entrainment mass flow rate and entrainment velocity. The low entrainment mass flow rate of $1 \mathrm{G} 2 \mathrm{~cm}$ channel could not penetrate through the swirl plume so that the centerline vorticity was not enhanced. However, the entrainment velocity can be expected to confirm the cross-sectional circulation and correspondingly, the 
cross-sectional vorticity, but its effect will be limited at the centerline due to the weakness of the radial velocity component there.

In addition, by narrowing the area of the gap, the inlet conditions (static suction pressure, velocity and mass flow rate) are raised (see Table III). Likewise, the entrainment ratio decreases (see Table IV). As result of the increase of inlet suction pressure, it is expected to increase the inlet axial velocity and consequently the mass flow rate. The increase of inlet mass flow rate can also be explained by the decrease of entrainment mass flow rate leaving more room for the inlet mass flow rate. In addition, due to the increase of inlet mass flow rate and decrease of entrainment mass flow rate, the entrainment ratio decreases substantially.

\section{Overall Effect of the Gap Width and the Number of Gaps}

The comparison between the results of the $2 \mathrm{G} 2 \mathrm{~cm}$ channel and the $1 \mathrm{G} 2 \mathrm{~cm}$ channel provides the opportunity to assess the simultaneous effect of increasing the number of the gaps and the total width of the gaps.

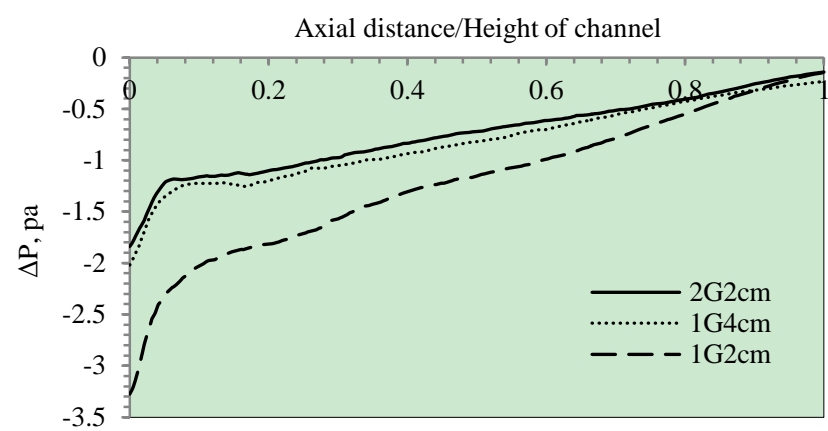

Fig. 5. Centerline pressure drop along the height for the three channels.

TABLE IV: ENTRAINMENT RATIO FOR THREE CHANNELS

\begin{tabular}{cl} 
Channel & Entrainment ratio \\
\hline $2 \mathrm{G} 2 \mathrm{~cm}$ & 29.6 \\
$1 \mathrm{G} 4 \mathrm{~cm}$ & 30.5 \\
$1 \mathrm{G} 2 \mathrm{~cm}$ & 16.8 \\
\hline \hline
\end{tabular}

By increasing the number of the identical gaps from one to two and increasing the total width of gap from $2 \mathrm{~cm}$ to $4 \mathrm{~cm}$, the total entrainment velocity along the gap declines (see fig. 3 ), and consequently the average entrainment velocity decreases (see Table II). However, the entrainment mass flow rate increases (see Table II). According to Fig. 5, the static pressure drop of $2 \mathrm{G} 2 \mathrm{~cm}$ is reduced compared to that for $1 \mathrm{G} 2 \mathrm{~cm}$ leading to a decrease in the pressure drop inside the $1 \mathrm{G} 2 \mathrm{~cm}$ channel and as a result, the entrainment velocity decreases for the $2 \mathrm{G} 2 \mathrm{~cm}$ channel. The increase of the total area of the gaps causes the increase of entrainment mass flow rate.

Increasing the number of gaps and the width of gap affects the central axial vorticity. The centerline vorticity increases by the increase in the number of gaps and the width of the gaps and the clear reason is the entrainment mass flow rate is introduced by two gaps at two sides of the channel and this increases the chance for sufficient penetration of ambient air to updraft air.

Due to the increasing of the number of the gaps and the total width of the gaps, the inlet static suction pressure decreases and this causes the direct reduction of inlet velocity and consequently the inlet mass flow rate decreases. Additionally, the entrainment ratio increases dramatically (see Table IV) due to the high total entrainment mass flow rate for the two gaps and the minimal inlet mass flow rate.

The overall trend of vorticity for the three channels is similar. The maximum centerline axial vorticity is near the base as shown in Fig. 4 and then the vorticity decreases as height increases. This can be explained by the entrainment velocity having an almost maximum constant value around the inlet which demonstrates the highest suction there and as a result generating maximal vorticity.

\section{CONCLUSION}

Three split channels with one and two side gaps have been modeled to assess the impacts of the number of the gaps and gap width on the characteristics of swirling flows within the channel. The results show that swirling flow can be generated using only one gap.

Increasing the number of the gaps without increasing the total width of the gaps increases swirling and reduces the inlet mass flow rate and entrainment velocity slightly which leads to a decrease in entrainment ratio. Increasing the gap width increases the entrainment ratio by increasing the entrainment mass flow rate. Increase of the number of identical gaps and the width of the gap decreases the entrainment velocity and the inlet mass flow rate but increases the entrainment mass flow rate and centerline vorticity. The entrainment ratio is the highest when the number of identical gaps increases.

It can be seen clearly that a split channel with two gaps can be used to improve fuel economy due to high strength swirl or as a split chimney to dilute pollution due to its high entrainment ratio. On the other hand, the split channel with one narrow gap can accelerate the existing plume due to the high suction at its inlet.

\section{REFERENCES}

[1] S. Al-Atresh, A. Sharifian, and A. Al-Faruk, "The effect of inlet velocity and temperature on the strength of the swirling induced by a spilt channel: A CFD approach," in Proc. 18th Australasian Fluid Mechanics Conference, Australia, 2012.

[2] Auto Electrical \& Mechanocal Repairs. (2014). Hiclone more power and economy with less pollution. [Online]. Available: http://www.kpauto.com.au/Products\%20-\%20Hiclone\%20-\%20Econo my.html

[3] S. R. Al Atresh, S. A. Sharifian, and B. Kueger, "Using a split chimney for dilution of exhaust pollution: A CFD approach," in Proc. 1th the International Conference on Fluid Dynamics and Thermodynamics Technologies, Singapore, 2012.

[4] T. Y. Palmer, "Laboratory and atmospheric vortex instabilities," The Journal of Weather Modification, vol. 11, no. 1, pp. 73-86, 1979.

[5] M. I. Hassan, A. Helali, and K. Saito, "Thermal and fluid dynamic structures of a laboratory-scale fixed-frame fire-whirl," in Proc. ASME International Mechanical Engineering Congress and Exposition, New York, 2001.

[6] M. I. Hassan, K. Kuwana, K. Saito, and F. Wang, "Flow structure of a fixed-frame type fire whirl," in Proc. 8th International Symposium On Fire Safety Science, U.S.A., 2005.

[7] K. Kuwana, S. Morishita, R. Dobashi, K. H. Chuah, and K. Saito, "The burning rate's effect on the flame length of weak fire whirl," in Proc. the Combustion Institute, 2011

[8] G. W. Zou, L. Yang, and W. K. Chow, "Numerical studies on fire whirls in a vertical shaft," in Proc.US-EU-China Thermophysics Conference-Renewable Energy (UECTC), Beijing, 2009. 
[9] Y. Huo, W. K. Chow, and Y. Gao, "Internal fire whirls induced by pool fire in a vertical shaft," in Proc. 8th Thermal Engineering Joint Conference (AJTEC2011), pp. T20034-T20034-6, U.S.A., ASME Press, 2011.

[10] K. Satoh and K. T. Yang, "Simulations of Swirling Fires Controlled by Channeled Self-generated Entrainment Flows," in Proc. 5th International Symposium on Fire Safety Science, 1997, pp. 201-212.

[11] B. Farouk, K. B. McGrattan, and R. G. Rehm, "Large eddy simulation of naturally induced fire whirls in a vertical square channel with corner gaps," American Society of Mechanical Engineers, Heat Transfer Division(HTD), 2000.

[12] W. Chow and S. Han, "Experimental data on scale modeling studies on internal fire whirls," International Journal on Engineering Performance-Based Fire Codes, vol. 10, no. 3, pp. 63-74, 2011.

[13] A. Sharifian, "The effect of wind speed on the performance of a split chimney," in Proc. 1th International Conference on Fluid Dynamics and Thermodynamics Technologies, Singapore, 2012.

[14] A. Sharifian and J. Hashempour, "Dilution of toxic gases and smoke emissions from residential chimneys using a low-cost cap," in Proc. the the 18th Australasian Fluid Mechanics Conference, Australia, 2012.

[15] C. Pozrikidis, Fluid dynamics: theory, computation, and numerical simulation, USA: Springer Verlag, 2009, ch. 5, pp. 222-223.
[16] J. Tu, Computational fluid dynamics: A practical approach, $1^{\text {st }}$ ed. Butterworth-Heinemann, U.S.A. \& U.K.: Elsevier, ch. 6, p. 258, 2008.

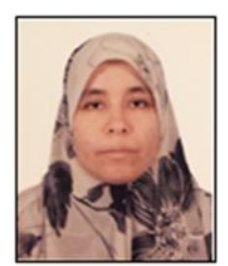

Safia R. Al Atresh was born in Libya on September 1, 1978. She received the B.A. in chemical engineering in 2001 and M.A. in chemical engineering in 2007 from University of Al- Mergeb in Al Khoums city in Libya. Her major field of study is fluid flow.

She worked as a teaching assistant from 2002 to 2007 and then worked as a lecturer at University of Al-Mergeb for one year. She has published three conference papers. Her current research interests are computational fluid mechanics and swirl flow.

Mrs. Al Atresh has been a member of the Australian Fluid Mechanics Society since 2012 . 